EXTENDED REPORT

\title{
Inequalities in access to knee joint replacements for people in need
}

\author{
P F K Yong, P C Milner, J N Payne, P A Lewis, C Jennison
}

Ann Rheum Dis 2004;63:1483-1489. doi: 10.1136/ard.2003.013938

See end of article for authors' affiliations

......................

Correspondence to: Dr Patrick F K Yong, Avon, Gloucestershire and Wiltshire Strategic Health Authority, Jenner House, Langley Park Estate, Chippenham, Wiltshire SN151GG, UK; pyong@ doctors.org.uk

Accepted 17 January 2004
Objectives: To quantify the effects of socioeconomic deprivation and rurality on evidence of need for total knee joint replacement and the use of health services, after adjusting for age and sex.

Methods: A random stratified sample of 15000 people aged $\geqslant 65$ years taken from central age/sex registers for the geographical areas covered by the previous Sheffield and Wiltshire Health Authorities. A self completion validated questionnaire was then mailed directly to subjects to assess need for knee joint replacement surgery and whether general practice and hospital services were being used. Subjects were followed up for 18 months to evaluate access to surgery.

Results: The response rate was $78 \%$ after three mailings. In those aged 65 years and over (with and without comorbidity), the proportion with no comorbid factors and in need of knee replacement was $5.1 \%$; the rate of need among subjects without comorbidity was $7.9 \%$. There were inequalities in health and access to health related to age, sex, geography, and deprivation but not rurality. People who were more deprived had greater need. Older and deprived people were less likely to access health services. Only $6.4 \%$ of eligible people received knee replacement surgery after 18 months of follow up.

Conclusions: There is an important unmet need in older people, with significant age, sex, geographical, and deprivation inequalities in levels of need and access to services. The use of waiting list numbers as a performance indicator is perverse for this procedure. There is urgent need to expand orthopaedic services and training.

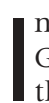
England, the NHS Plan and the National Priorities Guidance for the NHS for 2002/2003 have re-emphasised the political commitment to addressing health inequalities and inequities in accessing services. ${ }^{1}$ In 1997, the United Kingdom government set a target to reduce the number of people on waiting lists by 100000 , and the Patients' Charter provided for a maximum wait of 18 months for treatment. ${ }^{2} \mathrm{~A}$ recently published study calculated an annual underprovision of 26500 total knee replacement operations in England, although this figure does decrease when patient preference is taken into account. ${ }^{3}$ Health planners in all countries require data on health needs and service use for small areas to target services effectively. ${ }^{4}$ However, there has been only a limited number of previous studies assessing population needs for knee replacement surgery ${ }^{6}$ and none checking whether those in need received their knee replacement within the 18 month time limit for waiting.

The influence of socioeconomic deprivation on health in England is well established. ${ }^{78}$ However, the impact of rurality is under-researched. ${ }^{9}$ The original work on identifying an indicator of rurality for England and Wales using census data for 1971 and $1981^{10}{ }^{11}$ has been updated and expanded. ${ }^{12}$

The key characteristics of urban and rural deprivationsuch as low income levels, unemployment, and social exclusion-are the same. However, there are additional factors in the countryside that compound the problem. ${ }^{13-15}$ Sparsity of population means that access to services and the cost of living may be much greater. ${ }^{13-15}$ Car use may be a necessity in rural areas and can lead to considerable financial sacrifices in the absence of reliable public transport. ${ }^{13}{ }^{14}$ Much rural work is seasonal and low paid. There are often few work opportunities locally in rural areas for people with qualifications. The poor in rural areas are often geographically, culturally, and socially isolated. ${ }^{16}$

There have been attempts to develop a rural indicator of deprivation in England, but without success. ${ }^{14}$ The central essence of socioeconomic deprivation in urban and rural areas is low income. ${ }^{14}$ We have therefore chosen as our measure of socioeconomic deprivation the identification of individuals on low income.

Material deprivation is usually ascribed to an individual from their area of residence in health services research. ${ }^{17} 18$ However, it is better that an individual measure of material deprivation is used to avoid the ecological fallacy. It has been argued that income support dependency from the state is the closest measure of poverty in the United Kingdom, with the uptake of key means-tested welfare benefits being the best measure of individual material deprivation. ${ }^{13}$ We have used the uptake of means-tested benefits as our key individual measure of poverty.

The elderly often do not receive health services available to those who are younger.

The prevalence of need for knee joint replacement surgery is high among those aged 65 years and above. The procedure is an effective intervention for knee pain and disability secondary to arthritis, with improvements in all dimensions of health ${ }^{19}$ and good prosthesis survival rates. ${ }^{20}$ It has even been hailed by one editorial as the "joint of the decade." ${ }^{21}$ It performs strongly in economic evaluations when compared with other interventions. ${ }^{22-24}$

There is evidence in England to suggest that those most in need may be least likely to receive treatment. ${ }^{58}$ Very large geographical differences have also been found in the USA. ${ }^{25}$ Black Medicare recipients were less likely to receive total knee replacement than white recipients despite higher rates of knee osteoarthritis. ${ }^{26}$ A review of health services interventions ${ }^{28}$ undertaken for the Department of Health for England found few robust studies addressing such inequalities.

One recent study conducted by an international team of researchers in that field suggests that patients agree with health professionals that the relief of knee discomfort (pain 


\begin{tabular}{|c|c|c|}
\hline \multicolumn{2}{|c|}{ Description } & \multirow{2}{*}{$\begin{array}{l}\text { Census derived variable } \\
\text { Household/dwellings }\end{array}$} \\
\hline 1 & Occupancy rate & \\
\hline 2 & Commuting out pattern & $\begin{array}{l}\text { Percentage of residents in employment outside local authority } \\
\text { district }\end{array}$ \\
\hline 3 & Population: female 15 to 44 years & Percentage of total population \\
\hline 4 & Household amenities & $\begin{array}{l}\text { Percentage of households with exclusive use of fixed bath and } \\
\text { inside toilet }\end{array}$ \\
\hline 5 & Population density & Population/acre \\
\hline 6 & Occupational structure & Percentage employed in agriculture \\
\hline 7 & Population over 65 years & Percentage of total population over 65 \\
\hline 8 & $\begin{array}{l}\text { Distance from nearest } 50000 \\
\text { population urban node }\end{array}$ & $\begin{array}{l}\text { Distance in } \mathrm{km} \text { to nearest urban node }>50000 \text { population (not } \\
\text { from census data - calculated from National Reference Grid } \\
\text { maps) }\end{array}$ \\
\hline
\end{tabular}

and stiffness) and disability should be the main criterion for prioritising surgery..$^{29}$ In the present study we quantified the effects of rurality and socioeconomic disadvantage on previous evidence of need for knee joint replacement and the use of health services to meet that need, after adjusting for age and sex, in two areas in England. The two different areas offered a reasonable balance in terms of urban/rural communities and socioeconomic deprivation. We also assessed whether people identified through the survey as being in need of knee replacement underwent the procedure on the NHS within 18 months of receipt of their completed questionnaires, and whether there were any inequalities in this.

\section{METHODS}

The conduct of the study was governed by a written protocol that formed the basis of approval from the three local research ethics committees.

\section{Design}

The study was a cross sectional population prevalence survey, employing a self completed postal questionnaire. Our aim was to examine the uptake of key evidence based procedures for people in need. Hence, sample size was calculated by taking into account the prevalence of hip and knee disease in the population; the prevalence of cataract; an assumed response rate of $75 \%$; and stratification by deprivation.

The two areas differed in the way deprivation and rurality were spread. In a 5\% level, two sided test, we aimed for a power of 0.8 to detect a difference in rates of total knee replacement of a factor of 1.5 between high and low deprivation categories. Socioeconomic disadvantage for sampling purposes was assessed by using the Townsend index of material deprivation for area for residence. This is the best generally accepted single index of material deprivation in England, constructed from four variables derived from national census data. These variables are unemployment rate, housing tenure, care ownership, and household overcrowding. This score was allocated to all individuals in the

\begin{tabular}{lcl} 
Table 2 & Response rate by local authority \\
\hline Local authority & $\begin{array}{l}\text { Percentage } \\
\text { response }\end{array}$ & $\begin{array}{l}\text { Townsend index of material } \\
\text { deprivation (1991 census) }\end{array}$ \\
\hline Kennet & 80 & -1.39 \\
North Wiltshire & 81 & -2.99 \\
Salisbury & 82 & -1.09 \\
Swindon & 75 & -0.43 \\
West Wiltshire & 82 & -2.61 \\
Sheffield & 76 & 4.16 \\
Total & 78 & Not applicable \\
\hline
\end{tabular}

sampling frame. Scores were added for the smallest area level available. For Wiltshire, this was at ward level (mean population of 4500 people per ward) and for Sheffield it was at enumeration district (ED) level (mean population of 450 people per ED). Cumulative frequencies were compiled and the sample was divided into thirds. These three groups represented high, medium, and low levels of deprivation. In order to aid precision of the comparison between high and low deprivation categories, sampling was weighted towards subjects in these two categories, using proportions 2:1:2 for low, medium, and high deprivation levels.

Using this sampling scheme, it was calculated that a sample size of 15000 would be sufficient to achieve the power specification. The sample was weighted slightly in favour of the Wiltshire portion of the survey, because of differing numbers of people in the specified age group in two locations. Thus the sample was split between the two areas such that there were 7900 Wiltshire subjects and 7100 Sheffield subjects. The uptake of knee replacement by the study population was assessed by linking patient identifiers to NHS inpatient surgical data.

\section{Subjects}

Random subjects were drawn from the age/sex registers of the previous Wiltshire (7900) and Sheffield (7100) Health Authorities, stratified by disadvantage using the Townsend index of material deprivation (high, medium, low). Participants were selected from the high, medium, and low tertiles in the proportions $2: 1: 2$. Wiltshire has four district local authority councils (Kennet, North Wiltshire, South Wiltshire, and West Wiltshire) and one unitary local authority council (Swindon). Local authority areas are significant as they represent natural communities within administrative and political boundaries. All Wiltshire and Sheffield residents have access to primary, secondary, and tertiary care on the NHS. However, geographical access and the nature of community services may vary.

\section{Questionnaire}

Need for knee replacement in this study was determined by using an adapted version of the index of severity of osteoarthritis of the knee developed by Lequesne et al..$^{30}$ This instrument combines measurements of self rated knee pain, stiffness, and disability that have been validated against clinical diagnosis and decision making. The instrument has been used in a two stage process in another study in North Yorkshire, UK, to assess the need for knee replacement. ${ }^{31}$ It measures the discomfort and relative difficulties of subjects in performing activities of daily living such as using stairs, walking, getting up from a seat, and bending the knees. The index gives a score range of $0-24$ points. A threshold of 14 points was used as an indication of need for a specialist 
Table 3 Need for knee replacement and use of health services by age

\begin{tabular}{llllll}
\hline Age (years) & $\begin{array}{l}\text { Had knee replaced } \\
\text { (total population) }\end{array}$ & $\begin{array}{l}\text { Need knee replacing } \\
\text { (among population } \\
\text { with no comorbidity) }\end{array}$ & & \multicolumn{2}{l}{ Use of services by those in need (after exclusion of comorbidity) } \\
\cline { 5 - 6 } & $2(2$ to 3$)$ & $5(5$ to 6$)$ & $80(73$ to 85$)$ & $43(36$ to 51$)$ & Under GP care \\
$65-74$ & $4(3$ to 5$)$ & $10(8$ to 11$)$ & $73(66$ to 79$)$ & $34(27$ to 42$)$ & $14(9$ to 20$)$ \\
$75-84$ & $3(2$ to 5$)$ & $19(16$ to 23$)$ & $59(49$ to 69$)$ & $21(13$ to 31$)$ & $4(5$ to 14$)$ \\
$85+$ & & &
\end{tabular}

Values are per cent (95\% confidence interval)

$\mathrm{GP}$, general practitioner.

opinion regarding knee replacement, as this indicated extreme distress and disability. ${ }^{30}$ We repeated the analysis using a threshold of 11 points. The overall level of need was about $60 \%$ higher but led to the same conclusions regarding inequalities in health and treatment.

Questions taken from the North Yorkshire study ${ }^{31}$ were also included to assess the following:

- management of knee problems by general practitioner or hospital consultant, being on a hospital waiting list, and previous knee replacement;

- comorbidity, in order to identify those who would be unfit for surgery for other reasons;

- demographic details, such as sex and date of birth.

For determination of comorbidity, we used the approach detailed by Tennant et al, who carried out the North Yorkshire study. ${ }^{31}$ Comorbidity was determined from the questionnaire by asking respondents if they had ever been told by a doctor or health care professional that they had arthritis or rheumatism, Parkinson's disease, angina or heart disease, a stroke, a heart attack, severe bronchitis, or severe asthma. Obesity (as determined by body mass index over $30 \mathrm{~kg} / \mathrm{m}^{2}$, calculated from height and weight in the questionnaire) was also identified as a comorbid condition. All respondents indicating that they had any of the above conditions were automatically excluded. In addition, respondents were asked if they had any other illness or disability and were asked to give details of this. Answers in this section were then evaluated by medical staff to determine if the details given constituted comorbidity.

Deprivation was assessed by questions on individual or household receipt of means tested welfare benefits.

\section{Pilot}

An initial pilot study was conducted among an opportunistic sample of elderly people. Qualitative work with a group of elderly people attending a local day hospital confirmed the validity of the language used and a common interpretation of the questions asked. The questionnaire and the mailing process were then piloted on 100 subjects aged 65 years and above and randomly selected from the central age/sex register of the previous Wiltshire Health Authority. The format, questions, and approach were then modified accordingly.

\section{Mailing}

Three mailings were sent out, together with a letter from the relevant Director of Public Health for Wiltshire and Sheffield. The first mailing was sent out in November 1998. The first two mailings included a copy of the questionnaire and a reply paid envelope for return. The third mailing was only a reminder letter. Mailings were done at intervals of about three weeks.

\section{Data processing}

Data were double entered into a computer by a bureau. Queries from the bureau were resolved and the data subjected to consistency checks against the data model. A full audit trail was kept. There was only one data custodian, and patient identifiers were removed as soon as the unique NHS number was identified in order to safeguard the confidentiality of respondents.

\section{Analysis}

People with comorbid conditions (for example, marked obesity) and specific diagnoses (such as Parkinson's disease and stroke) which would probably preclude them from receiving knee replacement surgery were designated as being not eligible for it. These comorbid conditions were based on developmental work for the North Yorkshire study. ${ }^{31}$ Subjects were then categorised as follows:

- those in need and the need not met;

- those in need despite previous replacement surgery;

- those not in need who have had replacement surgery;

- those not in need who have not had replacement surgery.

Cloke's index at electoral ward level for the study area based on 1991 National Census data was used (O'Donohue D, personal communication) to help discriminate rural areas from urban ones. This consists of eight variables (table 1 ).

Multiple logistic regression analysis was used to predict the need for knee replacement as a function of age, sex, deprivation (in receipt of at least one means tested welfare benefit), health authority area of residence, and rurality (defined as a continuous variable based on Cloke's index for the area of residence). Also, the extent to which replacement surgery had been taken up in the 18 months following identification in our study was compared across the various population groups.

Table 4 Need for knee replacement and use of health services by sex

\begin{tabular}{|c|c|c|c|c|c|c|}
\hline \multirow[b]{2}{*}{ Sex } & \multirow{2}{*}{$\begin{array}{l}\text { Had knee replaced } \\
\text { (total population) }\end{array}$} & \multirow{2}{*}{$\begin{array}{l}\text { Need knee replacing } \\
\text { (among population with } \\
\text { no comorbidity) }\end{array}$} & \multicolumn{4}{|c|}{ Use of services by those in need (after exclusion of comorbidity) } \\
\hline & & & Under GP care & Under hospital care & On waiting list & Knee replaced \\
\hline Male & $3(2$ to 3$)$ & $5(4$ to 6$)$ & $76(67$ to 83$)$ & $40(31$ to 49$)$ & $12(7$ to 20$)$ & 7 (3 to 14$)$ \\
\hline Female & $3(3$ to 4$)$ & $10(9$ to 11$)$ & 72 (67 to 77 ) & 33 (28 to 39 ) & 12 (8 to 16$)$ & $6(4$ to 9$)$ \\
\hline
\end{tabular}


Table 5 Need for knee replacement and use of health services by deprivation

\begin{tabular}{|c|c|c|c|c|c|c|}
\hline \multirow{2}{*}{$\begin{array}{l}\text { Deprivation (in receipt } \\
\text { of means tested } \\
\text { benefits) }\end{array}$} & \multirow{2}{*}{$\begin{array}{l}\text { Had knee replaced } \\
\text { (total population) }\end{array}$} & \multirow{2}{*}{$\begin{array}{l}\text { Need knee replacing } \\
\text { (among population with } \\
\text { no comorbidity) }\end{array}$} & \multicolumn{4}{|c|}{ Use of services by those in need (after exclusion of comorbidity) } \\
\hline & & & Under GP care & Under hospital care & On waiting list & Knee replaced \\
\hline No & $3(2$ to 3$)$ & $6(5$ to 7$)$ & 77 (71 to 82$)$ & 37 (31 to 44 ) & 13 (9 to 18$)$ & $7(4$ to 11$)$ \\
\hline Yes & $3(2$ to 4$)$ & $14(12$ to 15$)$ & $68(61$ to 74$)$ & 32 (25 to 39$)$ & 10 (6 to 15$)$ & $6(3$ to 10$)$ \\
\hline
\end{tabular}

Values are per cent ( $95 \%$ confidence interval).

GP, general practitioner.

\section{Event linkage}

All people in the sampling frame, both responders and nonresponders, were followed up for 18 months by searching the relevant health authority datasets, and event linkage with NHS data was used to identify those who proceeded to surgery. However, this did not provide information on how many people might have moved out of the area or sought private health care. People who had only been put on a waiting list or had just consulted a specialist were not included because, given the level of need used in the questionnaire, these people would have been expected to have had their surgery within 18 months. Furthermore, the 18 month period was not measured from the time they first had their need, and it is not known how long people might have been waiting before that. The study population identifiers (NHS number, date of birth, sex, post code) were used to identify episode data in the health authorities databases that corresponded to the knee joint replacement procedures. Each episode record was coded to reflect the occurrence or not of an appropriate procedure. Where there were multiple episodes, these records were collapsed into a single composite record for each individual. These data were then linked to the "need" data by the study identification number. All personal identification data were then stripped from the records before analysis.

\section{RESULTS}

\section{Response}

The response rate was $78 \%$, with the more disadvantaged local authorities having slightly lower response rates (table 2 ). The response rate for those aged 65 to 74 years was $82 \%$, for those aged 75 to 84 years $77 \%$, and for those aged over 85 years $64 \%$.

\section{Representativeness}

The proportions by five year age bands in the sample and the populations studied were the same. In the sample, $43 \%$ of respondents were male- $2 \%$ more than in the study population. If men and women were equally likely to respond, the proportion of male respondents would be within 40 to $42 \%$ with a probability of 0.95 .

\section{Comorbidity}

Comorbid factors indicating that subjects would be unable to undergo surgery for knee replacement were present in 36\% of cases. Excluding these cases reduced the number of subjects in the analysis from 11214 to 7222 . There was almost total overlap between those designated as having comorbidity for either hip replacement or knee replacement. People with comorbid factors who were excluded were twice as likely to have an unmet need for knee replacement. A large percentage of these was excluded on the basis of obesity. A national report $^{32}$ stated that $19 \%$ of adults in England were obese and that this percentage increased with increasing age. We estimated the prevalence of obesity in our population to be about $24 \%$ using these national data. This would account for a significant proportion of survey respondents being excluded.

The approach used for determining comorbidity might have overestimated the number of people, as more minor forms of the comorbid conditions listed might not necessarily have precluded surgery. Consequently, this would have had the effect of underestimating the number of people eligible for surgery. Our event linkage data showed that some people with comorbidity received knee replacement surgery, indicating either that their comorbid condition was not sufficiently severe or had improved enough to allow knee replacement, or that their need was sufficiently severe for knee replacement to be the only option, despite comorbidity.

\section{Need}

Among the whole population aged 65 and above (including those with comorbidity and those without), the proportion of subjects who were in need of knee replacement and did not have comorbid factors was 5.1\% (95\% confidence interval (CI), $4.6 \%$ to $5.6 \%$ ). Our analyses will focus on those without comorbid factors. Of these, 5.3\% (95\% CI, $4.7 \%$ to $6.0 \%$ ) required both knee joints replacing. The unilateral need was very similar for both sides-left knee only, $1.3 \%$ ( $1.0 \%$ to $1.7 \%)$; right knee only, $1.4 \%$ ( $1.1 \%$ to $1.8 \%)$. In each age band, there was a much larger number of people needing knee replacements than people who had had previous surgery (table 3). Our estimate of the unmet need at December 1999 was about 4500 people in Wiltshire and 4600 in Sheffield.

There were 574 people in the study who were identified as being in need of knee replacement, after exclusion for comorbidity. The majority of these had not received the specialised care they needed. After follow up for up to 18 months, only 37 of those eligible $(6.4 \%$ (95\% CI, $4.3 \%$ to $9.2 \%)$ ) had received knee replacement surgery.

Table 6 Need for knee replacement and use of health services by rurality

\begin{tabular}{|c|c|c|c|c|c|c|}
\hline \multirow{2}{*}{$\begin{array}{l}\text { Rurality (tertiles from } \\
\text { Cloke's index) }\end{array}$} & \multirow{2}{*}{$\begin{array}{l}\text { Had knee replaced } \\
\text { (total population) }\end{array}$} & \multirow{2}{*}{$\begin{array}{l}\text { Need knee replacing } \\
\text { (among population } \\
\text { with no comorbidity) }\end{array}$} & \multicolumn{4}{|c|}{ Use of services by those in need (after exclusion of comorbidity) } \\
\hline & & & Under GP care & Under hospital care & On waiting list & Knee replaced \\
\hline Low & $3(3$ to 4$)$ & $9(8$ to 11$)$ & 76 (69 to 82 ) & 39 (32 to 47$)$ & 12 (7 to 18$)$ & $6(3$ to 11$)$ \\
\hline Middle & $2(2$ to 3$)$ & $8(6$ to 9$)$ & 76 (69 to 83 ) & $31(23$ to 40$)$ & 13 (8 to 20$)$ & 7 (3 to 12$)$ \\
\hline High & $3(2$ to 4$)$ & $7(6$ to 8$)$ & 66 (58 to 73 ) & $34(26$ to 42$)$ & $11(6$ to 17$)$ & 6 (3 to 11$)$ \\
\hline
\end{tabular}


Table 7 Need for knee replacement and use of health services by local authority

\begin{tabular}{|c|c|c|c|c|c|c|}
\hline \multirow[b]{2}{*}{ Local authority } & \multirow{2}{*}{$\begin{array}{l}\text { Had knee replaced } \\
\text { (total population) }\end{array}$} & \multirow{2}{*}{$\begin{array}{l}\text { Need knee replacing } \\
\text { (among population } \\
\text { with no comorbidity) }\end{array}$} & \multicolumn{4}{|c|}{ Use of services by those in need (after exclusion of comorbidity) } \\
\hline & & & Under GP care & Under hospital care & On waiting list & Knee replaced \\
\hline Kennet & 3 (2 to 6 ) & $7(4$ to 10$)$ & 69 (49 to 84 ) & 25 (11 to 45$)$ & $7(1$ to 24$)$ & $0(0$ to 13$)$ \\
\hline N Wiltshire & $2(1$ to 3$)$ & $7(5$ to 9$)$ & 78 (63 to 88 ) & 41 (27 to 57$)$ & 17 (7 to 32$)$ & 13 (5 to 26$)$ \\
\hline Salisbury & 4 (3 to 5 ) & $8(6$ to 10$)$ & $67(54$ to 78$)$ & 39 (28 to 52$)$ & 11 (5 to 22$)$ & $7(2$ to 16$)$ \\
\hline Sheffield & 4 (3 to 4$)$ & $9(8$ to 10$)$ & 78 (72 to 83 ) & 35 (29 to 42 ) & 11 (7 to 16) & $6(3$ to 10$)$ \\
\hline Swindon & $2(1$ to 3$)$ & $9(7$ to 11$)$ & 72 (61 to 82$)$ & 36 (25 to 48$)$ & $18(10$ to 30$)$ & $9(4$ to 18$)$ \\
\hline W Wiltshire & $2(1$ to 3$)$ & 5 (3 to 7$)$ & 53 (36 to 69$)$ & 21 (9 to 38 ) & $6(1$ to 21$)$ & $3(0$ to 15$)$ \\
\hline
\end{tabular}

Older people had much greater need, but those in need were a great deal less likely to be receiving care than younger people (table 3 ). Women were twice as likely to need knee replacement but equally likely to be receiving care (table 4).

Deprived people were more likely to need knee replacement (table 5). However, they were less likely to be receiving appropriate services.

People in rural areas had similar needs to those in other areas and were equally likely to be receiving care (table 6).

The greatest need for knee replacement was observed in the more disadvantaged local authority areas of Sheffield and Swindon. The proportion of people in need who were under GP care was low in West Wiltshire (table 7).

Multiple logistic regression analysis shows that age, sex, and poverty were all significant predictors of unmet need for knee replacement $(\mathrm{p}<0.001)$, with an increased likelihood of need for individuals aged over 75, for women, and for those in receipt of at least one means-tested benefit. These conditions have similar effects, each one independently increasing the probability of a subject being in need of a knee replacement by a factor of between 1.4 and 2.5. Health authority was also a predictor of need $(p=0.02)$, with a probability of need in Sheffield greater than that in Wiltshire by a factor of between 1.05 and 1.5. The multiple logistic regression analysis found no evidence of an association between rurality and the need for knee replacement after adjusting for the effects of age, sex, socioeconomic deprivation, and health authority area. Repeating the multiple logistic regression analysis without the health authority area term had no significant effect on this result.

\section{DISCUSSION}

\section{Principal findings}

The unmet need for knee replacement in those aged 65 years and above is relatively common at $5.1 \%$, after exclusion of those with comorbid conditions from the numerator. For the same (14 point) threshold on the Lequesne scale, ${ }^{30}$ our study showed a greater prevalence of need for knee replacement $(3.7 \% \vee 1.6 \%$ in those aged 65 to 74$)$ than in the North Yorkshire study. ${ }^{31}$ The estimate of prevalent need, with or without comorbidity, was also greater than found in the Somerset and Avon study, ${ }^{3}$ particularly for men (7.2\% v 2.2\% in men aged 65 to 74 and $8.9 \% v 6.0 \%$ in women aged 65 to 74). The differences in instrument, threshold, and approach used might account for the differences in prevalence between the various studies. Although the North Yorkshire study and our study used the same instrument and threshold (originally described by Lequesne ${ }^{30}$ ), it is possible that the two stage approach used in North Yorkshire excluded people who would have declared major symptoms if they had been asked more detailed questions. The Somerset and Avon study used an alternative instrument - the New Zealand score (which included a physical examination)-and this might have excluded some of the population identified in a pure questionnaire survey. However, the Avon and Somerset survey did show a greater prevalence of need than the North Yorkshire study, and this has not been fully explored in published reports. It is also possible that there is a true difference in the populations of the two areas or that this represents a temporal trend. Of the people who needed knee replacement, $67 \%$ needed both knees replacing. Of those aged over $85,14 \%$ either had a knee replacement or needed it.

After follow up for 18 months with event linkage, the proportion of people in need receiving knee replacement or revision surgery was very low (37 people, 6.4\%). The actual amount of utilisation of knee surgery by those in need is very low. No previous studies have been done to assess population need for knee replacement with subsequent follow up to determine whether people receive treatment. In a study done in Ontario, ${ }^{33}$ between $44 \%$ and $55 \%$ (depending on area) of people in need of hip or knee arthroplasty were definitely or probably unwilling to undergo surgery. The Somerset and Avon study $y^{3}$ found a $36 \%$ reduction in surgical need when incorporating patient preference. However, the methods used for assessing patient preference differ significantly between the two studies. Patient preference regarding surgery was not assessed in our survey but even with estimates of $55 \%$ of people in need not willing to have an operation, the percentage of uptake (14.3\%) among the remainder of subjects would still be very low.

The need for knee replacement increases markedly with age, but younger people needing knee replacement were more highly represented among patients under the care of their general practitioner or a hospital consultant or on a waiting list. This suggests there are age related inequalities in accessing health services

Women were twice as likely as men to need knee replacement. However, they were less likely to be under the care of their general practitioner or a hospital consultant and equally likely to be on a waiting list, although differences with regard to care were not statistically significant.

People who were socioeconomically deprived were about twice as likely to be in need of knee replacement as those who were more prosperous, but were less likely to be receiving services. Similar associations for knee replacements have been found in Medicare recipients. ${ }^{26}$ The Somerset and Avon study found a strong association between deprivation and self reported musculoskeletal disorders. ${ }^{34}$

The proportion on waiting lists varied among the local authorities, from $6 \%$ in West Wiltshire to $18 \%$ in Swindon, although the differences were not statistically significant.

There was no association between rurality and an increased need for knee replacement. After allowing for the level of need, people in rural areas were equally likely to be managed by their general practitioner or hospital consultant or to be on a waiting list as those in urban areas. This suggests, therefore, that any rural inequalities in accessing health services are small. ${ }^{35}$ Studies in the USA found that people in rural areas were more likely to receive knee replacement than those in urban areas. ${ }^{25}$ 
Older age, female sex, and being in receipt of at least one means-tested welfare benefit were all important independent predictors of an increased need for knee replacement. The degree of underuse of hip and knee arthroplasty has been reported to be more than three times greater in women than in men. ${ }^{36}$ In our study, women were about twice as likely as men to have unmet needs.

\section{Strengths and weaknesses of the study}

We used a validated self completed questionnaire to identify the need for knee replacement. This had previously been assessed against need as defined by clinical history taking, examination, and investigation. However, self completed questionnaires are not as accurate as diagnosis by a clinical expert. For example, they may overestimate the number of people requiring joint replacement by including a proportion of people in whom the severity of self reported symptoms differs from the opinion of clinicians following a more thorough assessment.

The response rate was reasonable at $78 \%$. However, there is still the possibility of non-response bias. Our estimates of need may be understated because non-responders in population surveys are usually more disadvantaged and tend to have more health problems. Apart from questionnaire data, we have all the register and postcode attributed data such as age, sex, post code, and Townsend material deprivation score on non-responders, as well as limited data on reasons for nonresponse (for example, deceased, moved out of area, and so on) available on request.

We used the Townsend material deprivation score to stratify the sampling. This is based on four census variables-the percentage of private households with more than one person per room, the percentage of private households with no car, the percentage of private households not owner occupied, and the percentage of residents eligible for employment who are unemployed. Using the Townsend area indicator for ascribing deprivation to individuals in our analyses would have missed a significant proportion of those receiving means-tested benefits.

There is an acceptance that the search for the ideal indicator of rurality is misplaced. ${ }^{12}{ }^{14}$ However, we used the indicator that is currently the most appropriate for health services research. ${ }^{12}$

We excluded about one third of people with an identified need because of comorbidities such as cancer and coronary heart disease. Again this might mean that our estimates are understated as not all self reported comorbidity would result in automatic exclusion for joint replacement given modern surgical and anaesthetic techniques. Also, this group of people have greater needs and are likely to add significantly to the total number of people eligible for surgery should a proportion of them be included. We also did not exclude patients who needed knee replacement but did not want it.

With regard to follow up, it was not possible to establish the number of people who had their knee replacement need met by private healthcare providers as these data were not available. In addition, no census data were available for people who had changed residence out of the area.

\section{Relation to other studies}

This is the first study in the United Kingdom highlighting the inequalities in the provision of knee replacement and the use of services. Hip replacement data from the same survey show similar inequalities. ${ }^{37}$ We used discomfort and distress as the sole health outcomes as these are the most important to patients. We did not include other outcomes that are dependent on the primary symptoms, because this might have given undue weight to the findings (as, for example, if four outcomes rather than two are reported as positive).

\section{Implications}

Our results are for people who have a threshold score of 14 or above and have extreme levels of discomfort and disability. If we set the threshold score at 11 to identify those who have severe levels of discomfort and disability, then the results indicate that there are very large numbers of people who need knee replacement (13.2\% of those aged 65 and above). Need among those precluded from treatment with a threshold score of 11 is $27.3 \%$. Currently, the waiting lists for orthopaedic services are the longest in England of all specialties in the NHS. This means that there is an urgent need to expand orthopaedic services and the training of orthopaedic surgeons. Strategic health authorities and primary care trusts in England are having their performance monitored publicly on the length and waiting times for key surgical procedures for residents. ${ }^{38}$ Clearly, if primary care were to identify all the unmet need for knee replacement currently and refer these cases to orthopaedic services, this would create even worse performance indicators for waiting times and numbers. However, paradoxically, despite the increase in waiting numbers, clinicians would be doing their job better as they would be offering a proven effective treatment to a greater number of people who would benefit. Consequently, the use of waiting list numbers as a performance indicator is perverse, as lower waiting list numbers in the context of a large burden of unmet need imply that many people eligible for treatment are not receiving it. At the same time, falling waiting list numbers create the illusion that the problem is being addressed, despite evidence of a large amount of unmet need in the community.

\section{Future research}

Our study demonstrates age, sex, geography, and deprivation inequalities in access to knee replacement surgery, and shows that only a small proportion of people in need actually receive surgery with relative speed. We need to understand why so many people in need of surgery are not receiving it. We need to know why so many people with needs are seeing their general practitioner or consultant orthopaedic surgeon and are not being put on a waiting list for knee replacement surgery. We also need to assess if there is a definite temporal increase in age/sex specific rates of need for knee replacement, and to explore whether there are inequalities in access to other evidence based procedures and services in England.

\section{ACKNOWLEDGEMENTS}

We thank Dan O'Donohue, Alan Tennant, Kevin Small, the previous Wiltshire and Sheffield Health Authorities, and the Rural Development Commission (now the Countryside Commission). We acknowledge funding by the previous Wiltshire and Sheffield Health Authorities and the Rural Development Commission (now the Countryside Commission).

\footnotetext{
Authors' affiliations

P F K Yong, Avon, Gloucestershire and Wiltshire Strategic Health Authority, Chippenham, Wiltshire, UK

P C Milner, P A Lewis, Department of Medical Sciences, University of Bath, Somerset, UK

J N Payne, School of Health and Related Research (ScHARR), University of Sheffield, South Yorkshire, UK

C Jennison, Department of Mathematical Sciences, University of Bath

\section{REFERENCES}

1 Department of Health. The NHS plan. London: HMSO, 2000.

2 Department of Health. Patient's charter. London: HMSO, 1991.

3 Juni P, Dieppe P, Donovan J, Peters T, Eachus J, Pearson N, et al. Population requirement for primary knee replacement surgery: a cross-sectional study. Rheumatology 2003;42:516-21.

4 Payne JN, Coy J, Patterson S, Milner PC. Is use of hospital services a proxy for morbidity? A small area comparison of the prevalence of arthritis, depression,
} 
dyspepsia, obesity and respiratory disease with in-patient admission rates for these disorders. J Epidemiol Community Health 1994;48:74-8.

5 Payne JN, Saul C. Variations in the use of cardiology services in a health authority: comparison of coronary artery revascularisation rates with the prevalence of angina and coronary mortality. BMJ 1997:314:257-61.

6 Holland R, Harvey I. Population needs assessment and knee replacement surgery. Rheumatology 2003;42:503-6.

7 Acheson, D. Independent inquiry into inequalities in health. London: HMSO, 1998.

8 Department of Social Security. Inequalities in health: report of a research working group. London: HMSO, 1980.

9 Fearn R. Rural health care: a British success or a tale of unmet need? Soc Sci Med 1987;24:263-74.

10 Cloke P. An index of rurality for England and Wales. Regional Studies 1977;11:31-46.

11 Cloke P, Edwards G. Rurality in England and Wales 1981: a replication of the 1971 index. Regional Studies 1986;20:289-306.

12 Harrington V, O'Donohue D. Rurality in England and Wales in 1991: a replication and extension of the 1981 rurality index. Sociologia Ruralis 1998;38:178-203.

13 Bruce A, Gordon D, Kessell J. Analysing rural poverty. Local Gov Policy Making 1996:1:16-23.

14 Noble M, Wright G. Identifying poverty in rural England. Policy Politics 2000;28:293-308.

15 ACRE. Rural poverty - the facts. ACRE, Cirencester, 1997.

16 Cloke P, Melbourne P, Thomas C. Lifestyles in rural England. A research report to the Department of the Environment, the Economic and Social Research Council and the Rural Development Commission. Salisbury: Rural Development Commission, 1994

17 Bradley PM. The choice of deprivation in measuring poorer health in Northamptonshire. J Epidemiol Community Health 1999:53:189-90.

18 Philimore P, Reading R. A rural advantage? Urban-rural health differences in northern England. J Public Health Med 1992;14:290-9.

19 Hawker G, Wright J, Coyte P, Paul J, Dittus R, Croxford R, et al. Health related quality of life after knee replacement. Results of the knee replacement patient outcomes research team study. J Bone Joint Surg Am 1998;80A:163-73.

20 Scuderi GW, Insall JN, Windsor RE, Moran MC. Survivorship of cemented knee replacements. J Bone Joint Surgery Br 1989;71B:798-803.

21 Moran CG, Horton TC. Total knee replacement: the joint of the decade. BMJ 2000;320:820.

22 Williams MH, Frankel SJ, Nanchahl K, Coast J, Donovan JL. Total knee replacement. In: Stevens A, Raftery J, eds. Health care needs assessment series, vol 1. London: Department of Health, 1994:524-90.
23 Hirsch HS. Total joint replacement: a cost-effective procedure for the 1990s. Med Health R I 1998;81:162-4.

24 Lavernia CJ, Guzman JF, Gachupin-Garcia A. Cost effectiveness and quality of life in knee arthroplasty. Clin Orthop 1997;245:134-9.

25 Peterson MGE, Hollenberg JP, Szatrowski TP, Johanson NA, Mancuso CA, Charlson ME. Geographic variations in the rates of elective total hip and knee arthroplasties among medical beneficiaries in the United States. J Bone Joint Surg Am 1992;74A: 1580-9.

26 Wilson MG, May DS, Kelly JJ. Racial differences in the use of total knee arthroplasty for osteoarthritis among older Americans. Ethn Dis 1994;4:57-67.

27 Katz BP, Freund DA, Heck DA, Dittus RS, Paul JE, Wright J, et al. Demographic variation in the rate of knee replacement: a multi-year analysis. Health Serv Res 1996;31:125-40.

28 NHS Centre for Reviews and Dissemination. Review of the research on the effectiveness of health service interventions to reduce variations in health. York: NHS Centre for Reviews and Dissemination, 1995.

29 Woolhead GM, Donovan JL, Chard JA, Dieppe PA. Who should have priority for a knee joint replacement? Rheumatology 2002;41:390-4.

30 Lequesne MA, Merry C, Samson M, Gerard P. Indexes of severity for osteoarthritis of the hip and knee. Scand I Rheumatol Suppl 1987;65:85-9.

31 Tennant A, Fear J, Pickering A, Hillman M, Cutts A, Chamberlain MA. Prevalence of knee problems in the population aged 55 years and over: identifying the need for knee arthroplasty. BMJ 1995;310:1291-3.

32 National Audit Office. Tackling obesity in England. London: HMSO, 2001

33 Hawker GA, Wright JG, Coyte PC, Williams JI, Harvey B, Glazier R, et al. Determining the need for hip and knee arthroplasty: the role of clinical severity and patients' preferences. Med Care 2001;39:206-16.

34 Eachus J, Williams M, Chan P, Davey Smith G, Grainge M, Donovan J, et al. Deprivation and cause specific morbidity: evidence from the Somerset and Avon survey of health. BMJ 1996;312:287-92.

35 Farmer JC, Baird AG, Iversen L. Rural deprivation reflecting reality. $\mathrm{Br} J \mathrm{Gen}$ Pract 2001;51:486-91.

36 Hawker GA, Wright JG, Coyte PC, Williams JI, Harvey B, Glazier R, et al. Differences between men and women in the rate of use of hip and knee arthroplasty. N Engl J Med 2000;342:1016-22.

37 Milner PC, Payne JN, Stanfield RC, Lewis PA, Jennison C, Saul C. Inequalities in accessing hip joint replacement for people in need. Eur J Public Health 2004; 14:58-62.

38 Department of Health. High level performance indicators. London: Department of Health, 1999. 